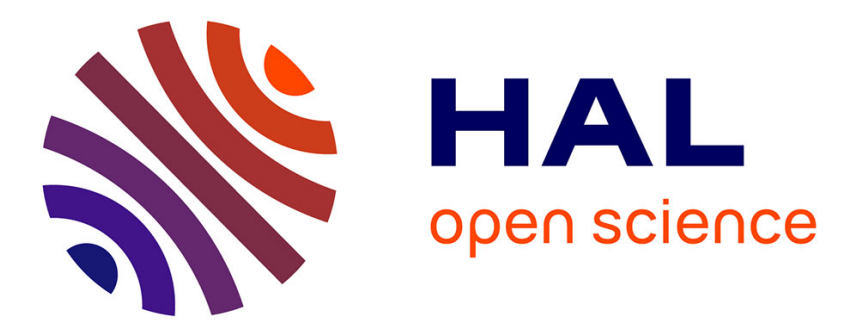

\title{
Alcool et grossesse : une recherche qualitative auprès de femmes enceintes
}

\author{
Julie Brahic, Olivier Thomas, Lionel Dany
}

\section{To cite this version:}

Julie Brahic, Olivier Thomas, Lionel Dany. Alcool et grossesse : une recherche qualitative auprès de femmes enceintes. Les cahiers Internationaux de Psychologie Sociale, 2015, 107, pp.403-434. 10.3917/cips.107.0403 . hal-01657564

\section{HAL Id: hal-01657564 \\ https://hal-amu.archives-ouvertes.fr/hal-01657564}

Submitted on 7 Dec 2017

HAL is a multi-disciplinary open access archive for the deposit and dissemination of scientific research documents, whether they are published or not. The documents may come from teaching and research institutions in France or abroad, or from public or private research centers.
L'archive ouverte pluridisciplinaire HAL, est destinée au dépôt et à la diffusion de documents scientifiques de niveau recherche, publiés ou non, émanant des établissements d'enseignement et de recherche français ou étrangers, des laboratoires publics ou privés. 


\title{
Alcool et grossesse : une recherche qualitative auprès de femmes enceintes
}

\author{
Alcohol and pregnancy: a qualitative research with pregnant women \\ Julie BRAHIC ${ }^{1}$, Olivier THOMAS ${ }^{1} \&$ Lionel DANY $^{2,3}$ \\ ${ }^{1}$ Association Méditerranéenne de Prévention et de Traitement des Addictions (AMPTA), 13002, \\ Marseille, France \\ ${ }^{2}$ APHM, Timone, Service d’Oncologie Médicale, 13385, Marseille, France \\ ${ }^{3}$ Aix-Marseille Université, LPS EA 849, 13621, Aix-en-Provence, France
}

La correspondance pour cet article doit être adressée à Lionel Dany, Laboratoire de Psychologie Sociale, Maison de la Recherche, Université d'Aix-Marseille, Maison de la Recherche, 29, avenue Robert-Schuman 13621 Aix-en-Provence cedex 1, France. Courriel : Lionel.Dany@univ-amu.fr

Le premier auteur a réalisé la recherche. Le troisième auteur a supervisé le projet de recherche, la phase de recueil et d'analyse. Les trois auteurs ont participé au travail de réflexion et de discussion des résultats, et ont rédigé l'article. 
Résumé : La consommation d'alcool pendant la grossesse constitue un enjeu de santé publique. Dans une perspective socio-représentationnelle, notre recherche visait à étudier les normes de consommations d'alcool pendant la grossesse. Soixante-quatre entretiens semidirectifs ont été réalisés auprès de femmes enceintes (Age moyen=28.9ans). Une analyse de contenu thématique a été réalisée sur l'ensemble des entretiens. Les représentations sociales concernant l'alcool pendant la grossesse sont particulièrement normatives. Cependant, nous pouvons observer la co-existence de règles générales (interdiction) et certaines cognitions conditionnelles (comportements autorisés sous certaines conditions). Le risque alcool pendant la grossesse s'exprime dans la confrontation de logiques explicatives qui reposent sur la prégnance de la responsabilité individuelle, sur la dimension sociale des conduites mais aussi sur le caractère conditionnel et contextualisé de la construction du risque.

Mots clés : Alcool, Représentations sociales, Grossesse, Femmes enceintes, Normes sociales.

\begin{abstract}
Alcohol consumption during pregnancy is a public health issue. From a sociorepresentational perspective, our research aims to study the norms of alcohol consumption during pregnancy. Sixty-four semi-structured interviews were conducted among pregnant women (average age of 28.9 years). The social representations concerning alcohol during pregnancy are particularly normative. However, we can observe the co-existence of general rules (prohibition) and some conditional cognitions (behaviors allowed under certain conditions). The 'alcohol risk' during pregnancy is expressed in the confrontation of explanatory logics based on the importance of individual responsibility, the social dimension of this behavior, but also on the conditional and contextual characteristics of the risk construction.
\end{abstract}

Keywords: Alcohol, Social Representations, Pregnancy, Pregnant Women, Social Norms. 


\section{Introduction}

\subsection{Alcoolisation au cours de la grossesse et risques associés}

Dans un contexte où l'alcool est largement consommé par les français et françaises ${ }^{1}$, il existe peu de données générales sur la consommation des femmes enceintes en France. Une enquête menée entre 2003 et 2004, auprès de 837 françaises enceintes (De Chazeron et al., 2008) a montré que plus de la moitié des françaises $(52,5 \%)$ reconnaissait avoir bu au moins une fois alors qu'elles étaient enceintes. D'après un sondage réalisé par l'INPES en 2004 et en 2007 sur les femmes enceintes, on peut observer que parmi celles qui buvaient de l'alcool (29\% en 2004 et $13 \%$ en 2007) la quasi-totalité avait une consommation inférieure à 2 verres par jour. La consommation d'alcool au cours de la grossesse chez les françaises diminue, ce changement se fait en général au premier trimestre (Waterson \& Murray-Lyon, 1989). La consommation d'alcool prend ainsi plus la forme d'épisodes ponctuels que celle d'une consommation régulière (au moins un verre plus d'une fois par semaine). On remarque toutefois que $13,7 \%$ d'entre elles, rapportent avoir consommé au moins cinq verres d'alcool durant cette période. Aujourd'hui, s'il existe une baisse de la consommation chez les femmes enceintes, elle reste toutefois présente, puisque $20 \%$ des femmes déclarent continuer à s'alcooliser durant la grossesse malgré la politique de prévention contre les dangers de la consommation d'alcool pendant la grossesse (Inserm, 2011). Une récente synthèse de la littérature sur l'usage de substances psychoactives durant la grossesse en France souligne que le fait d'avoir bu au moins une fois au cours de la grossesse varie de $12 \%$ à $63 \%$ selon les études (et régions), cet usage étant le fait de femmes plus âgées et appartenant plus souvent à un milieu social privilégié (cf. Dumas, Simmat-Durand, \& Lejeune, 2014). Par ailleurs, les épisodes d'alcoolisation ponctuelle importante (seuils $\geq 5$ ou $\geq 6$ verres selon les études) varient de $0,4 \%$ à $7,2 \%$ (Dumas et al., 2014).

La consommation d'alcool pendant la grossesse représente la première cause de handicap mental d'origine non génétique chez l'enfant en France. Qu'elle soit ponctuelle ou modérée, elle n'est pas anodine et peut entrainer des risques importants pour l'enfant à naître (INPES, 2006 ; Jacobson \& Jacobson, 2002) en étant responsable de diverses anomalies. Lorsqu'il y a des troubles graves ceux-ci sont irréversibles. L'ensemble des troubles causés par l'alcoolisation fotale - ETCAF (Lamblin, 2005) désigne toutes les répercussions, physiques, cognitives et/ou comportementales, qui peuvent être liées à une consommation d'alcool par la mère au cours de sa grossesse. Ces troubles incluent le Syndrome d'Alcoolisation Fotale (SAF), conséquence la plus importante pour l'enfant d'une exposition in utéro à l'alcool. Ce 
syndrome est facile à diagnostiquer, il entraine des anomalies de la face et du système nerveux central du bébé, des retards de croissance, une malformation de la boîte crânienne et de l'encéphale ainsi que des troubles nerveux. Le retard de croissance (dans $80 \%$ des cas) est le principal critère diagnostique du SAF. A long terme, cela se traduit par un retard mental, un déficit de l'attention, des difficultés à l'exécution de tâches motrices fines, une altération des capacités d'apprentissage et de mémorisation, voire l'apparition de psychoses (Astley et al., 1999 ; Famy et al., 1998 ; Roebuck et al., 1998 ; Streissguth et al., 1994). D’autres études ont montré des déficits chez les enfants ou adultes ayant un SAF (Kelly et al., 2000 ; Mattson et Riley 2000 ; Roebuck et al., 1999) en particulier plus de problèmes d'anxiété, de dépression et plus de difficultés dans les interactions sociales avec les autres (Thomas et al., 1998).

Les autres troubles causés par l'alcoolisation de la mère pendant la grossesse, sont appelés les Effets de l'Alcoolisation Fœtale (EAF). Ils peuvent être moins connus, parfois moins sévères et moins faciles à diagnostiquer, car ils apparaissent plus tardivement lorsque l'enfant grandi (tels que des troubles de l'apprentissage et/ou du comportement). On peut noter que la dysmorphie crânio-faciale est considérée comme spécifique de l'exposition à l'alcool in utero. Les troubles causés par l'alcoolisation fœtale sont estimés à 9\%o des naissances vivantes. L'incidence du SAF est estimée entre 0,5 et 3 cas pour 1000 naissances en France. Ainsi, une étude a montré que 700 à 3000 enfants sur les 750000 naissances annuelles, seraient concernés par un SAF grave (INSERM, 2001). D'après le ministère de la santé, il existerait 420000 personnes en France qui seraient atteintes du SAF. Il faut souligner que les répercussions d'une exposition prénatale à l'alcool sur le développement du bébé peuvent être très variables. Il dépend de la quantité d'alcool absorbée, de la durée d'exposition, de la façon de boire, du stade de la grossesse, des capacités métaboliques de la mère et de la sensibilité du bébé. Cependant, si l'absorption d'alcool est délétère pendant toute la période gestationnelle, le seuil en deçà duquel les risques sont nuls n'a jamais été mis en évidence, laissant place à l'interprétation de chacun, sur ce que l'on pourrait nommer une « consommation acceptable ». Toutefois, si aucun seuil de consommation sans risque n'a été identifié, toute consommation d'alcool pendant la grossesse peut être donc être considérée à risque (Dumas et al., 2014).

\subsection{Un enjeu de santé publique et de prévention}

$\mathrm{Au}$ regard de l'augmentation de la consommation d'alcool chez les jeunes femmes, des risques reconnus au cours de la grossesse et des consommations qui persistent chez les femmes enceintes, l'alcoolisation pendant la grossesse constitue un enjeu de santé publique. Les messages de prévention ont commencé à se diffuser, pouvant expliquer en partie la baisse 
de la consommation d'alcool pendant la grossesse ces dernières années. La campagne recommandant «Zéro alcool pendant la grossesse » s'inscrit dans ce contexte ${ }^{2}$. Entre 2004 et 2007, des sondages réalisés auprès du grand public, ont montré une augmentation de plus de $10 \%$ (de 21,2\% à 31,8\%) d'adhésion à l'opinion selon laquelle « il n'y a pas de consommation sans risque » pour les femmes enceintes (Guillemont \& Léon, 2008). Toutefois, sur cette même période on constate une augmentation de $1,2 \%$ (de 2,7\% à 3,9\%) sur l'évaluation qu'un verre par jour serait sans risque.

Il faut noter que les politiques de prévention mettent en avant d'autres comportements à risque pendant la grossesse, tels que la consommation de tabac. Dans un contexte où la consommation de tabac à tendance à augmenter y compris chez les femmes de 20-45 ans, on retrouve également une diminution d'une consommation de tabac chez les femmes enceintes. Elle est d'autant plus marquée aujourd'hui $(33 \%$ des femmes fumaient avant la grossesse, contre 17,1\% au cours de la grossesse) qu'en 2003 (sur 35,9\% des femmes qui fumaient avant la grossesse, $21,8 \%$ de ces dernières consommaient au cours de leur grossesse).

La perception des risques semble jouer un rôle important pour expliquer, d'une part, la part non négligeable des consommations déclarées, d'autre part, la façon dont les messages de prévention et/ou le risque alcool sont reconstruits. Par exemple, une étude réalisée au Québec (April et al., 2010), pour la majorité des femmes enceintes interrogées, ne pas consommer au cours de cette période va de soi. Cependant d'autres femmes continuent pour « le plaisir» et ne pas « s'empêcher de vivre ». On peut aussi voir dans l'enquête réalisée par Toutain (2009), réalisée sur des forums internet, que la recommandation de l'abstinence est généralement mal comprise par les femmes alors qu'une consommation raisonnable leur paraît envisageable. Dans cette enquête, $20 \%$ des internautes, évoquent les conséquences de la consommation d'alcool pendant la grossesse (SAF) et 6\% seulement les connaissent. Les autres femmes n'étaient pas «conscientes » des risques et pensent que la prise d'alcool ne représente pas un danger pour le bébé. Généralement ces dernières font davantage référence au savoir populaire ou à l'expérience qu'elles ont de la grossesse plutôt qu'aux messages de prévention. Elles citent également diverses sources d'informations telles que les émissions télévisuelles consacrées à la santé ou les campagnes de prévention, et accordent beaucoup d'importance à l'expérience de leur propre mère. Ce dernier point est important car différents travaux montrent que, concernant différents « risques » au cours de la grossesse (e.g., alcool, tabac), la transmission de mère en fille a autant de valeur que le discours médical (Abrahamsson, Springett, Karlsson, \& Ottosson, 2005 ; April et al., 2010 ; Guyon, Audet, April, \& De Koninck, 2007). Il faut également pointer le fait que les femmes enceintes sont davantage 
confrontées à « un message de modération, et non d'abstinence » (Dumas et al., 2014) de la part de leur entourage mais aussi de celle des professionnels de santé (Branco \& Kaskutas, 2001).

\section{Les représentations sociales comme « outil » d'analyse}

\subsection{L'alcool comme objet de sens commun}

L'alcool, en tant qu'objet social, comme le rapport que les individus et les groupes entretiennent avec sont le symbole d'une culture. La transmission véhiculée par la famille, les religions, les réseaux de pairs, mais aussi l'histoire, reflète les représentations positives et valorisantes liées à l'alcool. De ce fait elles sont plus nombreuses et plus facilement reconnues que les effets négatifs (Le Garrec \& Damour, 2007). Fortement ritualisé, l'alcool accompagne systématiquement les événements marquants de la vie sociale, qu'ils soient individuels ou collectifs. Si les conséquences de l'excès d'alcool (e.g., violences, conduite en état d'ivresse, maladies) sont dénoncées, sa consommation habituelle n'est pas véritablement mise en cause (Maresca et al., 2000).

L'alcool fait l'objet d'un véritable ancrage culturel dans notre société, il n'est pas un objet neutre. De nombreuses régulations sociales de ses usages existent et témoignent des normes qui entourent la consommation de l'alcool, celles du « bien-boire » comme celles associées à la régulation « genrée » des pratiques. Par exemple, l'alcoolisation féminine est plus sujette à la dénonciation collective (Baily et al., 1991 ; Rainaut, 2000 ; Ricciardelli, 2001). En ce sens l'alcool et les pratiques d'alcoolisation renvoient à des enjeux normatifs particulièrement saillants. En un sens l'alcool est et fait norme. Une norme sociale réfère à une règle de conduite dans une société ou un groupe social, notamment des manières d'agir. Les normes sociales définissent le domaine de l'action sociale en précisant ce que l'individu peut ou ne peut pas faire. Elles traduisent les valeurs et les idéaux dominants de la société ou du groupe (Demeulenaere, 2003). Elles garantissent les objectifs intergroupes et exercent en ce sens une certaine pression vers l'uniformité sociale. Ces pressions sociales vont amener l'individu à se rallier aux normes, en cherchant un accord entre lui et les autres dans le but d'être accepté par le groupe (Maisonneuve, 2000).

Les normes du boire ont fait l'objet de différents travaux et analyses (e.g., Ancel \& Gaussot, 1998 ; Barthes, 1957 ; Gaussot, 2004). Le savoir boire (i.e. entendu souvent comme le « bien boire ») est un facteur nécessaire à l'intégration sociale dans son double aspect d'accession à une identité sociale. Ce savoir boire repose encore sur une compétence singulière, qui sert à 
réguler sa consommation. La consommation excessive ou solitaire d'alcool est associée à une déviance (cf. Becker, 1985) et le consommateur fera l'objet d'une stigmatisation du fait de ses pratiques et de l'évaluation qui en découlera (e.g., Lo Monaco, Guimelli, \& Hidalgo, 2010). Les prescriptions dictées par les normes établies par le groupe de référence et/ou la société sont omniprésentes dans l'activité d'évaluation de l'individu, que le groupe soit présent et les rappelle explicitement, ou absent, et dans ce cas l'individu intériorise ces valeurs et normes (Hyman, 1942). La prévention contemporaine a participé de façon importante à cette «conscience de soi», les différentes campagnes de prévention invitant les récepteurs potentiels à se « subjectiver» (Berlivet, 2004).

Dans ce contexte de saillance normative des pratiques d'alcoolisation, il convient de rappeler une tendance prononcée dans la relation inégalitaire qui existe entre les hommes et les femmes dans la société quant à la permissivité des conduites d'alcoolisation. Durant les vingt dernières années, les comportements des femmes se sont modifiés ainsi que leurs rapports face à la consommation d'alcool. Comme le démontrent plusieurs études (Fillmore, 1987 ; Wilsnack et al., 1984), il y a aujourd'hui beaucoup plus de femmes, en comparaison au passé, qui consomment de l'alcool régulièrement. Toutefois, si l'espace de la consommation d'alcool s'élargit on peut noter que l'influence du genre perdure. «En effet, les bornes entre consommations féminines et consommations masculines ne s'abolissent pas : elles se déplacent. On assiste davantage à une mutation symbolique (l'acceptabilité et la possibilité de mettre en œuvre des pratiques) qu'à une réelle modification des pratiques du boire. Ainsi demeure une asymétrie entre hommes et femmes, qui souligne les contours d'un dimorphisme moral, et renforce l'idée d'une consommation d'alcool propre à chaque sexe » (Meidani, Dany, \& Welzer-Lang, 2005, p. 69).

\subsection{Construire le risque alcool : le regard des représentations sociales}

L'évaluation des risques n'est pas une activité réservée aux experts. Elle découle d'un jugement en relation directe avec la perception qui est faite du risque. Le risque existe ainsi en tant que risque réel, ou objectif, comme pour une alcoolisation au cours de la grossesse reconnue risquée pour l'enfant. Mais le risque existe aussi à travers les représentations que l'on s'en fait. En effet, le risque savant ou profane, est l'objet d'une construction éminemment sociale (Breakwell, 2007 ; Lupton, 1999) «qui implique un ensemble d'enjeux, à la fois individuels et sociaux qui d'une part s'actualise dans et par les situations auxquelles sont confrontés les individus et les groupes et d'autre part, mobilise des cadres de pensée préexistants au sein de la structure sociale » (Dany \& Apostolidis, 2012, p. 71). Autrement 
dit, la perception des risques va au-delà de l'individu et de son traitement «personnel » du risque, elle doit également être envisagée de manière collective et contextuelle.

La théorie des représentations sociales (RS) part du postulat « qu'il n’y a pas de coupure entre l'univers extérieur et l'univers intérieur de l'individu (ou du groupe). Le sujet et l'objet ne sont pas foncièrement distincts » (Moscovici, 1969, p. 9). Aussi, un objet n'existe pas en luimême, il existe dans un contexte actif, pour un individu ou un groupe et par rapport à eux, et c'est donc la relation sujet-objet qui détermine l'objet lui-même. Les RS regroupent ainsi un ensemble organisé et structuré d'informations, de croyances, d'opinions et d'attitudes (Abric, 1994) qu'un groupe social d'individus élabore à propos d'un objet, à partir de communications sociales. Ceci permet à l'individu ou au groupe, de donner du sens à ses conduites, de comprendre la réalité à travers son propre système de référence et donc, de s'y adapter et de s'y définir une place.

Les RS constituent « une forme de connaissance, socialement élaborée et partagée ayant une visée pratique et concourant à la construction d'une réalité commune à un ensemble social » (Jodelet, 1989, p.53). Ces représentations sont à la fois «le produit et le processus d'une activité mentale par laquelle un individu ou un groupe reconstitue le réel auquel il est confronté et lui attribue une signification spécifique »(Abric, 1987, p. 64). Les RS, souvent associées au « savoir naïf», ne sont pas des savoirs erronés. Il s'agit en fait d'une « connaissance autre que celle de la science mais qui est adaptée à, et corroborée par, l'action sur le monde » (Jodelet, 1989, p. 15). Autrement dit, l'étude des représentations permet de rendre compte à la fois du rapport que l'individu entretient avec «l'objet représenté », mais aussi de l'inscription de ce rapport dans un contexte social.

Des études ont pu montrer la pertinence de cette approche dans la construction du rapport à la maladie et aux risques (e.g., Jodelet, 1989 ; Morin, 1996). La construction du risque traduit un lien dynamique entre le sujet (individuel et collectif) et l'objet. Ce dernier constitue un support d'élaboration, d'actualisation de confrontation et d'expression des formes de connaissances visant à le circonscrire et à le maîtriser (Apostolidis \& Dany, 2012). L'objet risque, comme support des relations sociales, mobilise des modes de pensées contenus à la fois dans lui-même et dans le sujet qui pense. Ainsi, la construction sociale des risques sanitaires implique des dimensions symboliques, affectives, idéologiques et culturelles (Dany \& Apostolidis, 2012). Il est important de préciser que les processus décrits ici relèvent d'une véritable dynamique, les choses n'étant pas figées. Ainsi, les représentations tendent à évoluer, notamment au travers des échanges interindividuels, entraînant en conséquence des changements d'attitude (Rateau, 2000). Un autre point nous semble important. Les RS sont un 
ensemble organisé de cognitions qui sont prescriptives et/ou descriptives (Flament, 1994). L'aspect prescripteur d'une cognition renvoie à son caractère normatif (e.g., règles d'actions, « ce que l'on doit et peut faire »). Toutefois, comme le pointe très justement Flament (1994, p. 39), dans le domaine des RS, « les prescriptions sont massivement conditionnelles ». Cette propriété des cognitions prescriptives est particulièrement heuristique dans l'étude de la construction sociale du risque car elle subsume la coexistence de règles générales (e.g., en général il ne faut pas prendre ce risque) et de cognitions conditionnelles (e.g., dans telle situation particulière il est toléré de prendre ce risque). Cette propriété des cognitions de la RS nous semble pertinente pour étudier «l'édification» du risque (et ses différentes formes) auprès d'individus et la pluri-rationalité qui est en mesure de s'y exprimer.

\section{Problématique et objectifs}

Comme nous l'avons précisé en introduction, la consommation d'alcool au cours de la grossesse constitue un risque important pour le développement d'un ensemble de troubles notamment le Syndrome d'Alcoolisation Fœtale. Ce risque lié à l'alcoolisation durant la grossesse représente un enjeu de santé publique et fait l'objet d'actions de prévention reposant à la fois sur une communication grand public (e.g., campagne «Zéro alcool pendant la grossesse ») ; un dispositif d'information spécifique (e.g., étiquetage des bouteilles d'alcool avec un pictogramme spécifique) et une généralisation du recueil de l'information sur les consommations de psychotropes auprès du public cible via les professionnels de santé. Malgré ces différentes actions on peut observer que des consommations perdurent pendant la grossesse. Dans ce contexte, il nous semble pertinent et heuristique de nous interroger, sept ans après le développement d'une prévention spécifique concernant l'alcoolisation des femmes enceintes, sur l'appropriation des normes de consommations d'alcool pendant la grossesse et sur l'appropriation des recommandations qui y sont associées par les femmes concernées. Par ailleurs ce risque «particulier»- dans le sens où il actualise un risque habituel «le risque lié à l'alcoolisation dans une situation d'exception » (la grossesse) constitue un terrain propice à l'étude de la construction sociale du risque. Plus précisément, il nous semble intéressant d'appréhender la façon dont le risque alcool est construit pour soi et autrui dans un contexte de forte pression normative issue de l'environnement social (e.g., famille, entourage) et du discours de santé publique.

Ce projet d'analyse du risque alcool lié au contexte de la grossesse peut faire l'objet d'une analyse psychosociale (cf. Apostolidis \& Dany, 2012). Ce type d'analyse s'appuie sur un 
ensemble de présupposés (cf. Dany, 2015) : les comportements dit «à risque » comportent de façon intrinsèque des enjeux psycho-sociaux; les comportements «à risque » ne sont pas atomisés ; des régulations psycho-sociales influencent les choix opérés par les individus et la « rationalisation » de ces choix ; le risque fait l'objet d'une construction sociale qui organise le champ du travail sociocognitif des individus et des groupes; les normes sociales contribuent en tant qu'élément constitutif et constituant à cette construction sociale du risque ; la dimension identitaire est prépondérante dans la négociation du risque. Ces différents points d'analyse dressent les contours d'un projet d'intelligibilité des mécanismes liés à la négociation du risque par les individus et les groupes sociaux.

Notre projet a pour objectif d'étudier les mises en représentation ${ }^{3}$ profane du risque alcool dans le contexte de la grossesse. Plus précisément; l'objectif général de notre étude était d'étudier les représentations sociales du risque alcool chez des femmes enceintes. De façon plus spécifique, nous souhaitions (1) étudier la correspondance entre situation sociale (i.e. grossesse) et construction représentationnelle du risque alcool et des normes du boire en lien avec cette situation sociale; (2) mettre en évidence le rôle des dimensions normatives inhérentes à l'usage de l'alcool sur cette construction représentationnelle (e.g., rôle de l'entourage, des professionnels de santé, du contexte social) ; (3) appréhender les formes de catégorisation/singularisation entre substances psychoactives; (4) appréhender le recours potentiel à des cognitions conditionnelles dans la construction du risque alcool.

\section{Méthode}

\subsection{Une enquête par entretien de recherche}

Cette recherche s'inscrit dans une démarche compréhensive exploratoire. Nous visions l'interprétation de l'expérience subjective comme expérience sociale (Jodelet, 2006). Cette expérience subjective étant étudiée à partir de la théorie des représentations sociales. Nous avons retenu l'entretien de recherche comme outil afin d'explorer des faits qui concernent les systèmes de représentations (pensées construites) renvoyant aux idéologies, aux valeurs, aux croyances, aux images et au sens que les acteurs confèrent à leurs pratiques, ainsi que les pratiques sociales (faits expériencés) qui concernent davantage les enjeux, les expériences personnelles, les trajectoires et les processus. Par ailleurs, l'entretien constitue une méthode pertinente lorsque l'on souhaite cerner la singularité et la complexité des phénomènes, les situer dans leur dynamique psychologique et sociale et restituer leurs logiques internes sans les évaluer (Santiago Delefosse, 2001). 
Un guide d'entretien comprenant plusieurs parties a été élaboré (Tableau 1). Dans un premier temps, les personnes interviewées étaient amenées à s'exprimer sur la grossesse en général, la manière dont elle est vécue, ou encore les changements d'habitudes de vie mis en place par la personne enceinte. Dans un deuxième temps, le guide explorait les pratiques et les représentations d'une consommation d'alcool pendant la grossesse. Dans cette partie était abordé plus précisément le lien qu'entretient la femme enceinte avec l'entourage et les professionnels mais aussi le regard que porte la société sur les femmes consommant de l'alcool pendant la grossesse. Enfin, le guide abordait les messages de prévention entendus et la connaissance de la femme enceinte sur les risques d'une consommation d'alcool pendant la grossesse. Cette chronologie a été choisie parce qu'elle permet d'appréhender la trajectoire des changements de modes de vie de la femme enceinte, avant de cibler la question de l'alcool. De même, interroger les messages de prévention et le rapport aux professionnels de santé, permet d'échanger aussi bien avec des femmes consommatrices qu'abstinentes.

\section{Tableau 1}

\subsection{Analyse des entretiens}

Le corpus d'entretiens a fait l'objet d'une analyse de contenu thématique. L'analyse de contenu «est un ensemble de techniques d'analyse des communications visant, par des procédures systématiques et objectives de description du contenu des énoncés, à obtenir des indicateurs (quantitatifs ou non) permettant l'inférence de connaissances relatives aux conditions de production/réception (variables inférées) de ces messages »(Bardin, 1977, p. 47). Ce type d'analyse permet de rechercher les systèmes de sens et les modèles présents et de prendre en compte les dynamiques des représentations sociales ainsi que le rôle important qu'elles ont dans la production des énoncés.

Nous avons procédé à une catégorisation dont l'unité de base était le thème (univers discursif de l'énoncé). Pour réaliser cette tâche, nous avons procédé en deux étapes : le repérage des idées significatives et leur catégorisation. Nous avons réalisé à la fois une analyse thématique systématique de manière verticale (une analyse de chaque entretien), mais aussi une analyse horizontale sur l'ensemble des entretiens, où divers thèmes ont pu être dégagés, notamment en fonction de la problématique. Enfin une analyse quantitative (fréquence d'évocation des thèmes) a été élaborée sur chacun des thèmes afin de mettre en lumière le «poids » des thèmes dans le corpus d'entretien. L'ensemble des étapes a fait l'objet d'un travail commun et comparatif entre les différents chercheurs impliqués dans le projet. 


\subsection{Sélection et modalités de recrutement de l'échantillon}

La recherche a été réalisée auprès de différents établissements publics et privés de Marseille. Deux hôpitaux, deux cliniques et cinq centres de planification (PMI) ont participé au projet. Le choix de la diversité des établissements, s'est fait afin de garantir une hétérogénéité tant au niveau de la prise en charge proposée, que du public reçu. Après avoir fixé avec les professionnels de santé un calendrier de présence de la psychologue attachée à la recherche au sein des établissements, il lui a été possible de rencontrer les femmes enceintes, suivies dans ces structures.

Le recrutement des femmes à interroger, s'est fait avec l'aide des équipes. Ces dernières, composées de sages-femmes, infirmières, gynécologues, conseillères conjugales et secrétaires, ont orienté les femmes enceintes, après les entretiens qu'elles avaient avec les professionnels des structures, vers la psychologue sociale de la santé chargée de la recherche. Les professionnels ont proposé à toutes les femmes enceintes qui avaient un rendez-vous au sein de l'établissement, de participer à la recherche, les jours où la psychologue était présente dans les locaux, indépendamment de la consommation d'alcool des femmes ou d'autres caractéristiques. Il été proposé, aux femmes enceintes orientées vers la psychologue, de participer à une enquête sur les changements d'habitude de vie au cours de la grossesse en insistant sur l'anonymat et le volontariat.

\section{Résultats}

\subsection{Population}

$\mathrm{Au}$ total 64 entretiens ont été réalisés auprès de femmes enceintes. Les femmes qui ont participé à la recherche ont entre 16 et 41 ans et une moyenne d'âge de 28,9 ans. Les trois quarts de ces femmes sont en couple $(73,4 \%)$ et plus de la moitié d'entre elles ont déjà des enfants $(56,3 \%)$. Près d'une femme sur deux $(42,3 \%)$ en est au troisième trimestre de sa grossesse. La moitié de ces femmes $(51,6 \%)$ a un niveau d'étude équivalent ou inférieur au BEP/CAP et un tiers a fait des études supérieures (32,8\%). Enfin, la quasi-totalité des femmes vit à Marseille $(85,9 \%)$.

D'autre part, près de $60 \%$ des femmes ne boivent jamais d'alcool. Pour celles qui en boivent, cela reste des consommations peu fréquentes. Presque $20 \%$ des consommatrices, consomment une fois par mois ou moins, et $15 \%$ deux à quatre fois par mois. De même, la plupart des femmes interrogées ont l'habitude de prendre un ou deux verres par occasion $(23,4 \%)$, et pour 
celles qui consomment plus, cela ne dépasse jamais les quatre verres $(12,5 \%)$. Les raisons de ces consommations sont diverses. Presque la moitié boit de l'alcool pour fêter un évènement $(41,2 \%)$, et plus d'un tiers parce que cela leur procure un certain plaisir $(36,5 \%)$. Les femmes consomment également pour se détendre $(23,8 \%)$ ou pour pouvoir faire la fête $(15,8 \%)$. Enfin, l'alcoolisation envisagée à la suite de la grossesse selon les femmes, sera identique à celle d'avant $(85,9 \%)$, mettant à nouveau en avant, la grossesse comme une période d'exception.

\section{Tableau 2}

\subsection{Analyse du contenu des entretiens}

Quatre principaux thèmes sont ressortis de l'analyse. Le premier concerne les changements au cours de la grossesse (alimentation, consommation de produits psychoactifs, habitudes de vie). Le second, traite de la consommation d'alcool (représentations associées, risques perçus, connaissances des risques). Le troisième thème concerne les autres produits psychoactifs, en particulier le tabac. Enfin le quatrième porte sur le contexte social et le rapport à autrui (famille, entourage, professionnels de santé, société).

\subsubsection{Les changements face à la grossesse}

L'expérience de la grossesse amène des bouleversements chez la femme. Elle est marquée par des changements, une manière de vivre différemment son quotidien, notamment concernant l'alimentation, le travail, les habitudes de vie, mais aussi le comportement vis-à-vis de soi, des autres, de ses émotions. Ces changements peuvent être volontaires, sollicités voire « imposés ». Ce thème a été abordé par la totalité des sujets. En effet, celui-ci amorce le début de l'entretien et la rencontre avec les femmes. Les changements ont lieu dans une logique de préconisation, d'anticipation et de prévention. Ils témoignent de ce qu'il faut faire afin de maintenir une bonne santé pour soi mais aussi pour son enfant. Les changements sont envisagés pendant une période courte, d'exception sur le plan physiologique et sur le plan social.

«Ça demande de s'adapter, d'écouter ce qu'il se passe, ralentir. Des petites choses à petite échelle » $\left(8,21 \text { ans, seule, primipare, } 3^{\text {ème }} \text { trimestre, consommatrice }\right)^{4}$.

«Je fais plus de pauses, je suis plus fatiguée pour faire les choses. Je fais beaucoup de choses, dans les associations, je récupère des trucs, je m'occupe et je mets plus de temps à les faire. Mais bon pour moi je me repose déjà beaucoup, je ne me couche pas trop tard. Mon rythme de vie a changé » $\left(62,37\right.$ ans, conjoint, non primipare, $3^{\text {ème }}$ trimestre, consommatrice). 
Les changements en termes d'alimentation sont particulièrement prégnants $(80 \%$ des femmes interviewées y font référence). Pour $63 \%$ des femmes qui ont abordé la question, l'alimentation a été modifiée. Les raisons sont diverses: manger plus équilibré et plus sainement qu'auparavant pour la santé du bébé, manger différemment, à cause de la toxoplasmose ou du diabète, ou encore suivre «les fameuses envies de la femme enceinte » évoqués par $11 \%$ d'entre elles.

«J'ai repris le déjeuner, boire un peu plus, je faisais déjà des repas équilibrés » $(63,36$ ans, conjoint, non primipare, $3^{\text {ème }}$ trimestre, consommatrice).

«Tout faire pour sa santé, même pour l'alimentation. Je suis pas immunisée contre la toxoplasmose, bien faire cuire, bien laver, pour pas attraper des maladies, j'ai respecté l'hygiène alimentaire bien carrée, pas de médicaments etc., pour avoir la meilleure hygiène possible » $\left(34,29\right.$ ans, conjoint, primipare, $2^{\text {ème }}$ trimestre, non consommatrice).

Les changements provoqués par la grossesse sont majoritairement vécus comme des contraintes. Le discours des femmes désigne une volonté de revenir, après la grossesse, à la situation initiale afin de se « retrouver ».

«J'en ai marre, on se prive de tout, les sorties, je profite pas de ma journée comme je le veux, les magasins comme je le veux, c'est très long » $\left(38,19\right.$ ans, seule, primipare, $1^{\mathrm{er}}$ trimestre, non consommatrice).

« J'ai une vie pas marrante, il n'y a plus de plaisir et si peu de concret pour la grossesse. C'est une période de transition où il faut réapprendre à vivre autrement $\gg\left(3,30\right.$ ans, conjoint, primipare, $2^{\text {ème }}$ trimestre, consommatrice).

"J'ai un peu hâte quand même de retrouver mon espace, mon corps, et quelque chose de plus, le fait de plus fonctionner toute seule, je suis pas obligée d'être attentive tout le temps à l'autre, c'est pas évident, de mettre en place ça, de faire attention pendant 9 mois $\gg\left(8,21\right.$ ans, seule, primipare, $3^{\text {ème }}$ trimestre, consommatrice).

Certains changements désignent les modifications d'un point de vue social. Il existe une redéfinition des modes de participations sociales. On ne peut plus participer comme avant aux activités pendant la grossesse. Ainsi, on met en place des stratégies d'ajustement pour éviter certaines situations, certaines activités sociales (celles où on ne doit pas boire par exemple).

« Je suis extérieure aux soirées car les autres buvaient. Donc j'évite les autres pour pas sentir la privation et j'évite les situations où on boit » (3, 30 ans, conjoint, primipare, $2^{\text {ème }}$ trimestre, consommatrice).

"J'essaye d'inviter et d'éviter de me désocialiser. En fait, j'essaie de garder ma vie sociale qui est importante au moral, car pendant la grossesse on sent très bien que, y a des changements d'humeur » $\left(26,22\right.$ ans, conjoint, non primipare, $2^{\text {ème }}$ trimestre, non consommatrice).

Finalement, la grossesse amène la femme enceinte à vivre beaucoup de choses, très ambigües. La femme oscille entre émotions positives (42\%) et négatives (64\%), même s'il existe une prégnance à exprimer préférentiellement des émotions négatives versus positives. 


\subsubsection{L'alcool}

L'alcool, thème induit a été abordé par la totalité des sujets. Dans cette thématique, plusieurs idées sont ressorties telles que la consommation des femmes, les risques de l'alcool au cours de la période de grossesse et les risques concernant l'alcool en général. Dans un premier temps, concernant la consommation des femmes, on a pu relever celle du passé, celle d'aujourd'hui et celle de l'après grossesse. Près d'une femme sur deux (41\%) consommait de l'alcool avant sa grossesse : «comme tout le monde », «de temps en temps », "lors d'une occasion", "un peu de vin au cours des repas» ou encore "pour faire la fête, décompresser ». Les raisons évoquées par les personnes qui ne consommaient pas, sont des raisons liées aux croyances religieuses, à la santé, au goût et/ou à l'effet. Près de sept consommatrices sur dix (69\%) déclarent avoir arrêté de boire de l'alcool au cours de leur grossesse. Pour nombre de ces consommatrices, cet arrêt a un caractère « d'évidence » :

"J'ai arrêté du jour au lendemain, c'était évident, même si frustrant de dire non, à quelque chose que j'ai envie» (3, 30 ans, conjoint, primipare, $2^{\text {ème }}$ trimestre, consommatrice).

"C'est évident, pas besoin de lire, du moment que c'est mauvais pour nous c'est mauvais pour l'enfant» $\left(32,25\right.$ ans, conjoint, primipare, $3^{\text {ème }}$ trimestre, consommatrice).

«C'est dans la logique, comme le matin, on prend sa douche on se lave les dents » (21, 27 ans, seule, primipare, $2^{\text {ème }}$ trimestre, non consommatrice).

La raison principale de cet arrêt est bien entendu d'éviter les risques que l'alcool peut entrainer sur la grossesse, toutefois, cet arrêt ne se fait pas sans difficultés :

"C'est un peu dur quand même quand tout le monde boit avec ses amis, mais bon après on se dit on est enceinte, c'est plus difficile pour choisir à boire du coup » (32, 25 ans, conjoint, primipare, $3^{\text {eme }}$ trimestre, consommatrice).

«J'apprends sur moi, je remarque que je sens les verres d'alcool, pour avoir une sensation. Ça m'a étonné, car je n'ai jamais bu avec excès. Je me suis demandée si j'étais peut être une alcoolique à petite échelle, mais je crois que c'est surtout une dépendance » (3, 30 ans, conjoint, primipare, $2^{\text {ème }}$ trimestre, consommatrice).

Celles qui continuent de boire de l'alcool au cours de la grossesse (31\% des consommatrices d'alcool) boivent essentiellement quelques verres lors d'occasions « spéciales ».

" J'ai bu une coupe de champagne, à deux trois fêtes, ou gouter un peu de vin dans le verre de mon mari $\gg\left(9,34\right.$ ans, conjoint, primipare, $3^{\text {ème }}$ trimestre, consommatrice).

«De temps en temps oui, quand il y a des occasions, mais comme je suis enceinte c'est rare $»\left(60,28\right.$ ans, conjoint, non primipare, $3^{\text {ème }}$ trimestre, consommatrice).

Ces consommations s'accompagnent le plus souvent d'une forme plus ou moins prononcée de justification. 
«De temps en temps en connaissance de cause » $\left(1,37\right.$ ans, conjoint, non primipare, $1^{\mathrm{er}}$ trimestre, consommatrice).

«Je me disais la bière c'est bon pour la montée de lait, je me déculpabilisais comme ça. Moi ça me fait mon petit plaisir, et tout le monde est content » $(62,37$ ans, conjoint, non primipare, $3^{\text {ème }}$ trimestre, consommatrice).

La consommation envisagée après la grossesse a été abordée par un cinquième des femmes interrogées : 70\% d'entre elles sont des consommatrices en dehors de la grossesse. Certaines pensent qu'elles consommeront comme avant, d'autres au contraire pensent qu'elles consommeront différemment, puisque leur vie sera «quelque peu différente ». Cette reprise de consommation après la grossesse peut être retardée pour les femmes voulant allaiter $(13 \%$ d'entre elles).

«Je reprendrais mes soirées une fois par mois. Quand je bois c'est un apéro, et une bouteille de vin à plusieurs, un verre ou deux» $(26,22$ ans, conjoint, non primipare, $2^{\text {ème }}$ trimestre, non consommatrice).

« Je me languis après l'allaitement de boire un verre de rhum, je vais devoir attendre un peu » $\left(62,37\right.$ ans, conjoint, non primipare, $3^{\text {ème }}$ trimestre, consommatrice).

«Je dis souvent je vais me lâcher, me venger, après il y a l'allaitement mais non, d'autres préoccupations, responsabilités, je ferai la fête pas comme avant, les anciens plaisirs seront remplacés par des nouveaux» (3, 30 ans, conjoint, primipare, $2^{\text {ème }}$ trimestre, consommatrice).

Dans un second temps, les risques qu'entraine une consommation d'alcool ont été abordés par la totalité des femmes sur un versant descriptif. Pour les deux tiers (67\%) de ces femmes la consommation d'alcool de la mère pendant la grossesse, peut entrainer des risques pour le bébé. Les risques énoncés sont les malformations (16\%), les retards de développement (11\%), ou encore le manque d'alcool pour le bébé à la naissance (6\%). Les femmes qui abordent ces risques sont à la fois des consommatrices et des non consommatrices d'alcool.

«Petit poids, malformations. Pas possible d'avoir un bébé pour en faire un monstre » $\left(8,21\right.$ ans, seule, primipare, $3^{\text {ème }}$ trimestre, consommatrice).

«Avec tous les risques que ça génère au niveau du développement que ce soit cérébral ou quoi » (62, 37 ans, conjoint, non primipare, $3^{\text {ème }}$ trimestre, consommatrice).

"Peut-être l'enfant il va mourir si y a de l'alcool, ou sortir handicapé, mongolien, ou la trisomie $21 »\left(57,32\right.$ ans, conjoint, non primipare, $1^{\text {er }}$ trimestre, non consommatrice).

«Une fois qu'ils naissent, ils sont en manque, mais après je ne suis pas plus informée que ça» $\left(24,34\right.$ ans, conjoint, non primipare, $3^{\text {ème }}$ trimestre, non consommatrice).

Pour ces femmes, il existe un doute concernant la dose d'alcool possible que la femme a le « droit » d'ingérer au cours de la grossesse. Il leur est difficile de circonscrire le risque alcool. Certaines pensent que quelques verres sont acceptables, notamment lors des occasions et que seules les grosses consommations sont à proscrire. Finalement, c'est plus la quantité et non la fréquence qui apparait comme dangereuse. 
«Si tu bois de temps en temps je pense que c'est pas trop grave » $(48,25$ ans, conjoint, primipare, $2^{\text {ème }}$ trimestre, consommatrice).

"Il faut une grande quantité d'alcool pour que ça entraine des risques, sinon non je ne pense pas » (57, 32 ans, conjoint, non primipare, $1^{\mathrm{er}}$ trimestre, non consommatrice).

«Je sais pas si c'est pas un ou même dix, que c'est la même chose du moment qu'il y en ait $\gg\left(9,34\right.$ ans, conjoint, primipare, $3^{\text {ème }}$ trimestre, consommatrice).

« On sait pas vraiment s'il faut, faut pas, quel moment, on connait un peu le truc, c'est global » $\left(21,27 \mathrm{ans}\right.$, seule, primipare, $2^{\mathrm{ème}}$ trimestre, non consommatrice).

"Est-ce que c'est zéro, ou se limiter par rapport à sa consommation de base » (64, 30ans, conjoint, primipare, $3^{\text {ème }}$ trimestre, consommatrice).

Les risques liés à la consommation d'alcool ont été évoqués indépendamment de la période de la grossesse (47\%). Boire de l'alcool est risqué que l'on soit enceinte ou non, que l'on soit un homme ou une femme. Le risque alcool, sa perception, préexiste donc à la situation de grossesse.

«Pour nous déjà c'est pas bon, alors lui peuchère » (32, 25 ans, conjoint, primipare $3^{\text {ème }}$ trimestre, consommatrice).

« Déjà quand on est pas enceinte on doit faire attention à l'abus d'alcool » (19, 22 ans, conjoint, primipare, $3^{\text {ème }}$ trimestre, non consommatrice).

"On est bête quand même quand on est ado ou adulte, on se rend pas compte que ça peut agir sur la santé il faut attendre d'être enceinte pour s'en rendre compte » $(34,29$ ans, conjoint, primipare, $2^{\text {ème }}$ trimestre, non consommatrice).

Sept femmes sur dix $(71 \%)$ estiment que la population est au courant des risques liés à la consommation d'alcool au cours de la grossesse, il s'agirait d'un savoir partagé. Ce résultat témoigne de la diffusion de la norme d'abstinence telle qu'initiée par la campagne «zéro alcool pendant la grossesse ».

" Aujourd'hui tout le monde est au courant, aujourd'hui tout le monde le sait, je pense qu'il n'y a personne sur terre qui sait qu'il faut pas boire enceinte, que c'est grave pour la santé $d u$ bébé » $\left(9,34\right.$ ans, conjoint, primipare, $3^{\text {ème }}$ trimestre, consommatrice).

«Je pense que c'est connu, mais il y en a qui suivent pas les règles, mais normalement c'est dans la logique, comme le matin, on prend sa douche on se lave les dents. Je pense que même un enfant dirait c'est pas bon de boire et fumer» $(21,27$ ans, seule, primipare, $2^{\text {ème }}$ trimestre, non consommatrice).

L'attitude envers les femmes qui ont un rapport pathologique à l'alcool (dépendantes et/ou alcooliques) a été abordée par près de deux femmes sur dix qu'elles soient consommatrices ou non. Ces dernières ne légitiment pas la consommation de ces femmes mais l'alcoolisme vient signifier l'impossibilité de contrôler les consommations. A ce titre, les femmes interviewées évoquent également une logique du «moindre mal» qui vise à tolérer la consommation d'alcool sous prétexte que cette consommation (souhaitée modérée) serait moins nocive que l'arrêt de la consommation lui-même et ses conséquences (e.g., stress). 
«Ils le savent, une grosse fumeuse je comprends que ça soit difficile à arrêter, mais une fumeuse occasionnelle non, elle doit s'arrêter. Un alcoolique ne peut rien y faire » (32, 25 ans, conjoint, primipare, $3^{\text {ème }}$ trimestre, consommatrice).

"Après pour ceux qui ont une addiction, c'est peut être mieux de boire un verre par jour que pas boire du tout, je sais pas, plutôt que de se chopper un stress pas possible, comme la cigarette $»\left(64,30\right.$ ans, conjoint, primipare, $3^{\text {ème }}$ trimestre, consommatrice). «Après ça dépend, des femmes qui buvaient avant, sont dans des pathologies d'alcoolisme, elles ont conscience du mal qu'elles font mais se font déjà du mal à ellesmêmes, c'est difficile aussi, je suis pas sévère avec, ce sont des personnes qui sont dans des pathologies, dans une addiction et on sait à quel point c'est dur d'en sortir seul, surtout suivant l'entourage qu'on a, les alcooliques sont souvent entourés d'autres alcooliques » $\left(62,37 \mathrm{ans}\right.$, conjoint, non primipare, $3^{\text {ème }}$ trimestre, consommatrice).

\subsubsection{Les autres produits psycho-actifs}

$\mathrm{Au}$ cours des entretiens, les femmes ont abordé les autres produits psycho actifs, tels que la cigarette, le cannabis ou encore la cocaïne et les risques liés à ces produits, et plus globalement les représentations qui en émanent. Parmi elles, 31\% d'entre elles sont des consommatrices de tabac. Au cours de leurs grossesses, certaines se sont arrêtées de fumer dès le début (un tiers) pour d'autres, l'arrêt s'est fait au fur et à mesure de la grossesse.

«Je fume pas. J'ai arrêté quand j'ai appris que j'étais enceinte, je ne consommais pas beaucoup avant, je commençais, et quand j'ai su j'ai arrêté de suite » (45, 22 ans, conjoint, primipare $2^{\text {ème }}$ trimestre, non consommatrice),

"Avant ça rythmait les pauses dans mon travail d'éducatrice, le café, la cigarette. J'ai gardé une par jour de 5 à 7 mois, mais dans l'ambiance de travail, pas quand j'étais en repos ou week-end. Depuis que je suis en arrêt ça me fait plus du tout envie, ça s'est régulé tout seul, déjà je n'avais pas envie de fumer. Je l'appréciais, même si je culpabilisais de voir mon gros ventre avec ma cigarette, je le faisais avec quelques collègues qui étaient plutôt cools, qui me faisaient déculpabiliser, je ressentais vraiment ce besoin de décompresser, je me disais "tant pis je fume je fume quoi », puis ça s'est vite arrêté » $\left(62,37\right.$ ans, conjoint, non primipare, $3^{\mathrm{e} m e}$ trimestre, consommatrice).

Au contraire, l'arrêt ne s'est pas mis en place pour $22 \%$ des fumeuses. Les raisons sont diverses. Pour certaines cela représentait une trop grande difficulté, pour d'autres l'arrêt n'a pas été réellement questionné.

«La cigarette c'est difficile, l'alcool c'est, comme je suis pas encore alcoolique, je peux arrêter. Non la cigarette c'est plus difficile. Je fume 15 cigarettes à presque un paquet par jour » (4, 28ans, conjoint, primipare, $3^{\text {ème }}$ trimestre, consommatrice).

«Là j'essaie d'en consommer encore moins, j'essaie de pas dépasser les cinq, voire les sept, ce qui est pas évident. Malheureusement, c'est pas grand-chose, mais c'est le peu dont j'ai besoin. Je culpabilise car j'ai un petit dans le ventre et je me dis c'est pas bon pour lui, mais quand l'envie est trop forte vous regardez plus rien" (24, 34 ans, conjoint, non primipare, $3^{\text {ème }}$ trimestre, non consommatrice).

«Je continue de temps en temps, quand j'en ai envie, pour ne pas être frustrée » $(1,37$ ans, conjoint, non primipare, $1^{\mathrm{er}}$ trimestre, consommatrice). 
Le stress ressenti constitue un vecteur important pour justifier le fait de ne pas arrêter ou ne pas arrêter complètement la consommation de tabac pendant la grossesse. En filigrane, on retrouve la même logique du «moindre mal » évoqué pour les personnes alcooliques.

« Je consommais un paquet par jour, là j’ai diminué je suis à deux trois cigarettes, je tente d'arrêter. Je trouve que je me débrouille pas mal quand même, bon si ça pouvait être aucune, se serait bien, mais disons que je suis assez stressée quand même, je suis assez nerveuse et ça me calme de fumer, donc c'est dur d'arrêter aussi » (14, 29 ans, conjoint, non primipare, $2^{\text {ème }}$ trimestre, non consommatrice).

«Pas envie de se contraindre totalement et être stressée, pas bien, speed. Je préfère doser petit et penser que ça va bien se passer » (12, 27 ans, conjoint, primipare, $3^{\text {ème }}$ trimestre, consommatrice).

«Ma collègue qui n'arrivait pas à arrêter, on lui a dit vaut mieux pas arrêter, et continuer à fumer quelques-unes de temps en temps sinon vous allez être stressée à cause du manque, elle ne lui a pas dit tabac zéro, mais alcool zéro » (34, 29 ans, conjoint, primipare, $2^{\text {ème }}$ trimestre, non consommatrice).

Par ailleurs, la consommation de cannabis a été évoquée par $16 \%$ des femmes interrogées.

Parmi elles, certaines étaient des consommatrices, et ont traité le produit sous l'angle de la dépendance.

"C'est différent car je n'arrive pas à m'arrêter, je suis dépendante. Mais j'ai déjà réussi à diminuer, je suis passée de quinze à cinq/dix joints par jour. C'est dur, mais pas en prendre ce ne serait pas possible, je serais pas bien, énervée contre tout le monde, sans qu'on m'ait fait quoi que ce soit » (2, 19 ans, chez ses parents, primipare, $1^{\mathrm{er}}$ trimestre, consommatrice).

"Je fumais beaucoup de joints par jour, j'ai fini par arrêter mes consommations pour la santé de ma fille, mais le cannabis a pris plus de temps, elle avait un périmètre crânien petit, on m'a dit que ça venait peut être de là, alors j'ai d'un coup arrêté » (19, 22 ans, conjoint, primipare, $3^{\text {éme }}$ trimestre, non consommatrice).

\subsubsection{Contexte social et rapport à autrui}

Seul un tiers des femmes (37,5\%) nous disent que la consommation d'alcool pendant la grossesse n'a pas été abordée avec leur entourage, le plus souvent parce que l'alcool ne fait pas parti de leur contexte de vie. Toutefois, le plus souvent on peut relever que l'environnement proche de la femme enceinte commente, témoigne, donne des conseils. Il fait partie de la vie de la femme et de sa grossesse. De ce fait, concernant l'alcool, l'entourage donne aussi son avis.

"L'entourage peut se manifester sur ça, c'est aussi son rôle, après ça dépend de l'entourage. » (26, 22 ans, conjoint, non primipare, $2^{\text {ème }}$ trimestre, non consommatrice). "Si mon copain était cool, qu'il s'en fichait, j'aurai bu un verre par mois en dehors des mois les plus craignos, mais là c'est évident de pas boire car il est protecteur, angoissé de la santé. Donc je lui ai montré que j'étais hyper vigilante avec ça» $(3,30$ ans, conjoint, primipare, $2^{\text {ème }}$ trimestre, consommatrice). 
Le plus souvent l'entourage tient un discours favorable aux messages de prévention et conseille de «ne pas boire pendant la grossesse ». L'entourage constitue par ses actions et interventions un « rappel à la norme ».

"C'est évident pour les gens que je ne bois pas. Si je trempe mes lèvres pour sentir le gout, les gens bloquent "Qu'est-ce qu'elle est en train de faire? », du coup je me justifie... Je suis surprise de la réaction des gens » (3, 30 ans, conjoint, primipare, 2 ème trimestre, consommatrice).

«On me dit directement « toi tu ne bois pas », quand la personne a la bouteille, on me dit «bon toi non ». C'est une évidence. Quelqu'un qui m'inviterait je le remettrai à sa place, c'est incohérent $\gg\left(26,22\right.$ ans, conjoint, non primipare, $2^{\text {ème }}$ trimestre, non consommatrice).

"C'est connu qu'il ne faut pas boire pendant la grossesse, donc ils ne vont pas proposer, si on refuse ils ne vont pas insister. Quand ça se voyait pas peut être qu'ils ne disaient pas comme ça je sais plus, mais là il y en a qui disent "ah mais toi je te propose même pas », une remarque comme ça» $\left(33,27\right.$ ans, conjoint, primipare, $1^{\mathrm{er}}$ trimestre, consommatrice).

Seule une minorité de femmes (17\%) explique que l'entourage a un discours contraire aux messages de prévention, il aurait plus tendance à « autoriser» la femme à se faire plaisir avec un verre d'alcool de manière occasionnelle :

«Souvent les gens disent «tu peux prendre un petit fond»»(5, 30ans, conjoint, non primipare, $3^{\text {ème }}$ trimestre, consommatrice).

«J'ai des copines qui boivent de temps en temps, c'est plutôt cool sans excès. Je pense que quand les gens voient qu'il y a une conscience de ça, voilà quand les gens voient que c'est pas tous les jours, même pas toutes les semaines, qu'il y a une attention particulière, que tout a été mis au ralenti, personne ne dit rien » $(8,21 \mathrm{ans}$, seule, primipare, $3^{\text {ème }}$ trimestre, consommatrice).

"Moi on m'a embêtée pour me dire "vas-y un petit verre ça va pas faire de mal, il va pas non plus sortir de là alcoolisé, handicapé, les gens ne comprennent pas, ma bellefamille n'a pas compris pourquoi du jour au lendemain j'arrêtais, je leur expliquais, ils me regardaient en me disant "à l'époque on se prenait pas autant la tête », mais comme je leur ai dit c'est pas se prendre la tête, moi je suis au courant d'un problème, je suis informée, j'ai pas envie même si au départ c'était difficile, boire un verre de champagne ou un verre de vin rouge avec du fromage, c'est délicieux, 9 mois où on porte quelqu'un dans notre ventre, pour sa santé on a pas le droit d'avoir une hygiène de vie comme ça» $\left(34,29 \mathrm{ans}\right.$, conjoint, primipare, $2^{\mathrm{ème}}$ trimestre, non consommatrice).

Les femmes interviewées évoquent la modification de la société et l'évolution du regard face aux pratiques de consommation. Les femmes mettent en avant la société hygiéniste dans laquelle on évolue, pour tempérer le risque. Actuellement, les normes et le regard portés sur nos comportements ont changé, et en particulier pour tout ce qui concerne la consommation de substances psycho-actives. En effet, certaines femmes se basent sur l'expérience des générations précédentes pour parler des produits et de sa consommation pendant la grossesse : 
«À l'époque on ne se prenait pas autant la tête» (34, 29 ans, conjoint, primipare, $2^{\text {ème }}$ trimestre, non consommatrice).

"Les anciens me disent " moi quand j'étais enceinte je buvais je prenais l'apéritif et regarde mes enfants ils sont en bonne santé » $\left(58,33\right.$ ans, conjoint, non primipare, $3^{\text {ème }}$ trimestre, consommatrice).

«Les vidéos de Noël avant, où ils sont tous dans la même pièce en train de fumer, les enfants au milieu, mais bon personne n'était informé, nous si, après chacun ses choix» (33, 27 ans, conjoint, primipare, $1^{\mathrm{er}}$ trimestre, consommatrice).

Concernant les professionnels de santé, la quasi-totalité des femmes (97\%) a abordé le lien entretenu avec eux, ainsi que le rôle qu'ils ont dans leurs interventions pendant la grossesse. On remarque qu'ils sont un moyen pour les femmes d'avoir accès à des messages de prévention (intervention directe du professionnel, distribution de prospectus).

«Parce que c'est la gynécologue qui nous a dit: zéro alcool pendant la grossesse, seulement pour ça» $\left(11,34\right.$ ans, conjoint, primipare, $3^{\text {ème }}$ trimestre, consommatrice).

"L'alcool aussi elle m'a dit que c'était pas bon pour la grossesse, mais je bois pas d'alcool je sais que c'est pas bon. Ma gynécologue m'a dit qu'il pouvait devenir dépendant » $\left(60,28\right.$ ans, conjoint, non primipare, $3^{\text {ème }}$ trimestre, consommatrice).

" Le médecin m'a donnée un papier sur ce qu'on peut faire ou pas, alimentation, alcool... et sur les risques de maladies qu'on peut attraper» (4, 28 ans, conjoint, primipare, $3^{\text {ème }}$ trimestre, consommatrice).

Pour $72 \%$ des femmes, au moins un des professionnels de santé leur a demandé si elle consommait de l'alcool et/ou du tabac. La question était posée de manière systématique et la réponse simplement recueillie. Cette situation peut faire l'objet d'un calcul en terme de présentation de soi pour les femmes.

«Ils m'ont demandée, c'était des questions, si je fume, bois, mais ils n'ont pas parlé des risques, je pense parce que je consomme pas » $\left(22,25\right.$ ans, conjoint, non primipare, $1^{\mathrm{er}}$ trimestre, non consommatrice).

«Ils m'ont demandée, m'ont expliquée les risques qu'il y avait, peut être que pour ma troisième grossesse c'est ce qu'on m'avait dit sur l'alcool qui m'a fait un déclic et que je consomme plus» $\left(43,35\right.$ ans, conjoint, non primipare, $1^{\text {er }}$ trimestre, non consommatrice).

"Ici elles m'ont dit «ça va, pas de cigarette pas d'alcool? " sur la liste j'avais mis si sur la cigarette, j'avais mis un ou deux, ils vous le demandent à un moment donné, ils cherchent pas à savoir si ça a évolué ou pas puis ils font pas de commentaires sur ça, des fois on se dit qu'est-ce qu'ils vont en faire, quel jugement ils vont porter, par contre sur l'alcool j'ai mis non, histoire de pas rentrer dans les détails, oui j'en ai consommé, je me dis si je mets "oui " ils vont penser quoi, que je consomme tous les jours de l'alcool, mais vu qu'ils en demandent pas plus pour la cigarette, je me dis si je mets oui, ça va faire les alarmes partout, si j'ai bu trois verres de cidre sur la grossesse, c'est pas la peine, on sait pas les répercussions que ça peut avoir. C'est dommage parce que le dossier est pas vraiment ok, puis pleins de choses peuvent se passer entre temps, ce n'est pas la peine de générer des choses pour rien, ça fait ça en moins à se soucier au cours de la grossesse » $\left(62,37\right.$ ans, conjoint, non primipare, $3^{\text {ème }}$ trimestre, consommatrice). 
Le rapport aux professionnels de santé active de façon importante les enjeux du « dire » ou du « taire ». Face aux professionnels de santé, il semble difficile de dissimuler ou de ne pas dire la vérité. C'est un « devoir de vérité » qui s'exprime.

" C'est la première personne à qui on s'adresserait, c 'est l'instinct de mère, on en parle on est obligé, c'est logique » (4, 28 ans, conjoint, primipare, $3^{\text {ème }}$ trimestre, consommatrice).

«Ben je vais pas mentir, franchement oui je leur aurai dit, parce que après c'est pour mon bébé, après ils ont besoin de le savoir, après peut être qu'ils auraient pu m'aider » $\left(14,29\right.$ ans, conjoint, non primipare, $2^{\text {ème }}$ trimestre, non consommatrice).

«Très honnêtement, j'ai dit à la sage-femme que j'avais bu deux verres de champagne et un petit peu de vin » $\left(12,27\right.$ ans, conjoint, primipare, $3^{\text {ème }}$ trimestre, consommatrice).

\section{Discussion}

L'analyse des données a permis de mettre en évidence la correspondance entre la situation sociale spécifique que représente la grossesse et la construction représentationnelle du risque alcool et les normes afférentes. La période de grossesse est une période de changements, à la fois biologique, physique et social. Il semble important de pointer qu'il s'agit d'un temps de transition entre deux statuts, d'une période « liminale » (cf. Van Gennep, 1909) $)^{5}$ se déroulant sur un temps défini mais elle ne peut être envisagée comme une période de rupture totale. Par « rupture », nous voulons souligner que le temps de la grossesse ne constitue pas une période d'exception exclusive mais qu'elle s'apparente davantage à une période de rupture dans la continuité. Par ailleurs, ces changements pourraient donner l'apparence de s'effectuer « spontanément» ou «naturellement», toutefois, une analyse plus fine permet de montrer qu'ils opèrent sous le prisme de l'influence du contexte socio-normatif.

Plus précisément, et concernant la consommation d'alcool, on constate au cours de la grossesse, une maximisation des normes préexistantes, qui régulent le rapport que les femmes entretiennent avec l'alcool dans un contexte social donné. L'identité de mère se superpose à celle de femme, de fait, ce n'est plus uniquement l'analyse du rapport de la femme à l'alcool ou de l'individu à l'alcool qui devient opérant, mais l'analyse conjointe du rapport femmealcool et mère-alcool. Finalement, le fait que la femme s'apprête à devenir mère (ou être mère de nouveau) suppose qu'elle va devoir faire face à des responsabilités parfois nouvelles pour les primipares dans un contexte social prescripteur de diverses formes de régulations sociales de la maternité (Lapierre \& Damant, 2012; Menuel, 2012). Comme en témoignent les personnes interviewées on assiste durant cette période à une focalisation plus importante sur les règles d'hygiène de vie et les règles socio-sanitaires en termes d'entretien de soi de la part 
des femmes et de leur environnement social. Ces règles ne sont pas nouvelles car pour nombres d'entre-elles elles sont déjà admises voire mises en œuvres avant la grossesse.

Les propos des femmes montrent qu'il existe une ambivalence au niveau de la perception des informations sur l'alcoolisation pendant la grossesse et des risques qu'elle entraine. Si toutes les femmes déclarent qu'il est risqué de boire de l'alcool pendant la grossesse, la moitié d'entre-elles ne peut définir les risques ou les expliquer. En effet, ces risques ne sont pas toujours connus ou compris. Peu de femmes ont déjà entendu le terme de syndrome d'alcoolisation fœtale et une minorité d'entre elles est capable d'en définir les conséquences. Ces dernières ont été évoquées notamment par déduction d'expériences et de discours entendus. Pendant la grossesse, l'alcoolisation quotidienne ou massive est perçue comme dangereuse car elle est associée à un risque direct pour l'enfant. La connaissance de la norme de non consommation est très présente, comme dans d'autres études (April et al., 2010 ; Maalouf et al., 2011 ; Menuel, J. 2011).

Les risques évoqués renvoient principalement au handicap, à la malformation. Ces contenus rejoignent pour partie les savoirs scientifiques tout en ce centrant sur les conséquences les plus sévères (INPES, 2006 ; Kelly et al., 2000 ; Lamblin, 2005). De fait, la consommation d'alcool de la femme enceinte est considérée comme « inacceptable » et « choquante » pour les femmes interviewées et plus globalement pour la société telle que perçue par ces femmes. Les risques perçus concernant l'alcoolisation pendant la grossesse concernent donc les risques biomédicaux mais on peut ajouter à ceux-ci des risques plus sociaux relevant des jugements sociaux et moraux qui s'appliquent à la femme enceinte qui consomme de l'alcool. Il s'agit là d'un risque identitaire du fait de la stigmatisation de ce comportement.

On retrouve toutefois des avis partagés de la part des femmes sur la dose acceptable de consommation possible. Cela peut être notamment lié à la quantité préconisée d'alcool absorbée pendant la grossesse qui n'est pas toujours précise. En effet, le seuil n'est pas connu ni annoncé de manière claire, laissant ainsi un espace possible au doute. De fait, pour certaines femmes, consommer un verre de temps en temps n'est pas systématiquement condamnable et ne donne pas lieu à une dévalorisation de leur image, car le risque perçu pour l'enfant est faible. Cette négociation du risque, comme cela a été observé dans d'autres travaux (e.g., April et al., 2010 ; Maalouf et al., 2011 ; Toutain, 2009), est plus présente chez les consommatrices.

Les RS concernant l'alcoolisation en cours de grossesse sont massivement « teintées » d'une composante normative. Dans ce cadre, le contexte social et le rapport à autrui (famille, 
entourage), constituent des points nodaux de la mise en représentation du risque alcool chez les femmes interviewées. La famille et/ou l'entourage rappelle la norme, en évoquant les règles de conduites à tenir, les risques qu'entraîne l'alcool. Ces normes circulent dans la société comme au niveau des réseaux amicaux et familiaux, c'est à ce titre qu'elles ont une influence sur les comportements des femmes enceintes. C'est par les jeux de normalisation explicites et implicites, auxquels contribuent la famille et l'entourage, que se mettent en place les transformations des représentations et des comportements, et finalement l'intériorisation de la norme (Fassin \& Memmi, 2004).

Un point important - du fait de la spécificité de l'objet étudié et sa place dans le champ sociosanitaire - concerne le rôle perçu des professionnels de santé. Les femmes interrogées déclarent que ces professionnels les questionnent sur leurs habitudes de vie, leurs consommations de tabac et/ou d'alcool. Cependant, si la consommation n'est pas perçue comme «problématique » par les professionnels (reste à définir ce qui peut être déclaré ou perçu comme problématique dans cette situation), ces derniers ne vont pas plus loin dans leurs démarches. On peut souligner que la question posée sur la consommation d'alcool «buvezvous de l'alcool? » sous-entend généralement pour les femmes interrogées, une consommation d'alcool de manière régulière voire quotidienne (INPES, 2006). Ceci peut ainsi expliquer pourquoi les femmes répondent majoritairement qu'elles ne consomment pas lorsque cette question est posée. La mesure du risque alcool par les professionnels de santé s'apparente à une routine. Ce questionnement quasi-standardisé superficiel (tel qu'il est décrit par les femmes interviewées) pourrait d'une certaine manière renforcer le phénomène de « biopolitique délégué » (Memmi, 2004) qui peut conduire à une non-déclaration ou une sous-déclaration de comportements jugés inappropriés et relevant de la responsabilité personnelle ${ }^{6}$.

Par ailleurs ce questionnement induit pour partie les réponses acceptables et/ou possibles. En effet, les campagnes de prévention pour la santé, "poussent en fait les membres du public à se définir en déclarant leur identité de non-fumeur, de "buveur modéré », etc... (Berlivet, 2004, p.65). En d'autres termes, la réponse à cette question n'est pas le seul produit de la rencontre avec le professionnel dans le cadre de la situation thérapeutique. Elle est issue, pour partie, du cadre social préexistant qui délimite les «champs du possible » par rapport à ce type de questionnement. Autrement dit, les femmes interrogées savent quoi répondre pour donner l'image attendue voire nécessaire. On peut penser que ce dispositif de questionnement (celui qui se déroule dans la rencontre avec le professionnel de santé) ne permet pas nécessairement de recueillir une information au-delà de ce qu'il est convenable de dire. Le 
caractère systématique et injonctif de la question ne permet pas d'individualiser l'approche des comportements à risques potentiels.

Comme nous l'envisagions, l'analyse des entretiens a permis de faire émerger, à la fois, des formes de catégorisation/singularisation entre substances psycho-actives mais aussi le recours à des cognitions conditionnelles dans la construction du risque alcool et du risque tabac. Malgré les connaissances profanes ou scientifiques que les femmes ont des risques qu'entrainent des produits tels que l'alcool ou la cigarette, les pratiques de consommation pendant la grossesse peuvent perdurer. L'alcool et le tabac sont différenciés mais on peut observer des logiques de construction du risque similaire. Par exemple, la consommation de tabac a souvent été abordée de façon concomitante au discours produit sur la consommation d'alcool. A ce titre, on peut évoquer qu'il n'est pas rare que les individus, quel que soit le contexte dans lequel ils sont interrogés, effectuent un travail de construction représentationnel sur une substance en faisant appel aux autres substances afin d'en circonscrire les spécificités par des jeux de catégorisation/singularisation (cf. Apostolidis \& Dany, 2012; Dany \& Apostolidis, 2002). On peut relever que comparativement à l'alcool, il existe une certaine acceptabilité de la consommation de tabac, qui est perçu comme un produit moins néfaste et de fait plus acceptable pour la plupart des femmes. Selon ces dernières, l'arrêt du tabac au cours de la grossesse, provoquerait un stress qui ne serait pas favorable au développement du bébé. Ce processus consisterait à penser un risque non acceptable habituellement selon les normes de la société (fumer pendant sa grossesse), comme un risque qui serait plus acceptable, comparativement à un autre risque (stress provoqué par l'arrêt et ses conséquences possibles sur l'enfant) envisagé comme ayant un niveau de dangerosité plus élevé. On se trouve face à des logiques de pensées conditionnelles (cf. Flament, 1994) à travers lesquelles des évènements ou pratiques qui ne devraient pas être mises en œuvre peuvent l'être et surtout peuvent être justifiées voire légitimées. Ces processus de rationalisation puisent dans un savoir de sens commun qui «légitime» certains risques (e.g., consommation modérée de tabac) comparativement à d'autres perçus comme étant plus nocifs (e.g., stress provoqué par l'abstinence). Ainsi qu'il s'agisse de l'alcool ou du tabac on se trouve en présence de la coexistence de règles générales (e.g., en général il ne faut pas fumer ni boire d'alcool pendant la grossesse) et de cognitions conditionnelles (e.g., du fait du stress potentiel provoqué par l'abstinence et ses répercussions possible sur l'enfant il est toléré de fumer quelques cigarettes, voire de consommer de l'alcool si on est alcoolique). Nous ne sommes pas en présence d'un déni du risque (cf. Peretti-Watel, 2005), dans le sens où le risque ne serait pas 
(re)connu. Dans l'une ou l'autre des situations (alcool et/ou tabac) les femmes interrogées sont au courant que la situation entraîne des risques. On observe chez elles, consommatrices et non consommatrices, une (re)construction des niveaux de risque à travers une grille de lecture qui puise dans des savoirs sociaux, des représentations, des croyances. Cette (re)construction ne se fait pas uniquement à travers des pratiques de consommations, ni à travers l'analyse « objective » des dangerosités associées à ces pratiques, mais bien à travers des connaissances et logiques sociales qui pourraient tout à fait être sollicités en dehors de la période de la grossesse mais qui s'actualisent aussi au cours de cet événement. On se trouve face à une négociation du risque (Dany \& Apostolidis, 2002 ; Dany, 2014) qui rend compte des dynamiques interpersonnelles et psychosociales en jeu dans la construction du risque. Les individus «négocient» le rapport qu'ils entretiennent avec le comportement à risque considéré afin de concilier croyances sociales, normes et prescriptions, responsabilité personnelle, identité sociale et contrôle de la situation.

Pour conclure, nous dirons que la tension entre «l'individuel » et le «collectif » se manifeste de façon composite pour les femmes enceintes lorsqu'il s'agit de penser le risque alcool. Cette tension s'exprime en premier lieu dans la construction sociale du risque alcool qui rend compte des processus éminemment sociaux de cette construction (i.e. les contextes sociaux et culturels comme cadres dans lesquels le risque est produit, le «risque » comme valeur où figurent des choix sociaux) et de processus individuels de (re)définition des risques en lien avec des enjeux identitaires. Elle s'exprime également dans la confrontation de logiques explicatives des conduites à risques qui reposent sur la prégnance de la responsabilité individuelle, sur la dimension sociale de ces conduites mais aussi sur le caractère conditionnel et contextualisé de la construction du risque.

\section{Bibliographie}

- Abrahamsson, A., Springett, J., Karlsson, L., \& Ottosson, T. (2005). Making sense of the challenge of smoking cessation during pregnancy: a phenomenographic approach. Health Education Research, 20, 367-378.

- Abric, J.-C. (1994). Pratiques sociales et représentations. Paris : PUF.

- Abric, J.-C. (1987). Coopération, compétition et représentations sociales. Cousset : Delval.

- Ancel, P., \& Gaussot, L. (1998). Alcool et alcoolisme: Pratiques et représentations. Paris: L'Harmattan.

- April, N., Audet, C., Guyon, L., \& Gagnon, H. (2010). Représentations sociales et consommation d'alcool pendant la grossesse. Drogues, Santé et Société, 9, 17-48. 
- Astley, S. J., Magnuson, S. I., Ommell, L. M., \& Clarren, S. K. (1999). Fetal alcohol syndrome: changes in craniofacial form with age, cognition, and timing of ethanol exposure in the macaque. Teratology, 59, 163-172.

- Bailly, R. C., Carman, R. S., \& Forslund, M. A. (1991). Gender differences in drinking motivations and outcomes. Journal of Psychology, 125, 649-656.

- Bardin, L. (1977). L'analyse de contenu. Paris : PUF.

- Barthes, R. (1957). Mythologies. Paris : Éditions du Seuil.

- Becker, H. S. (1985). Outsiders. Paris : Métaillié.

- Berlivet, L. (2004). Une biopolitique de l'éducation pour la santé. La fabrique des campagnes de prévention. In D. Fassin \& D. Memmi. (Eds.). Le gouvernement des corps, cas de figure (pp. 3775). Paris : Editions de l'EHESS.

- Breakwell, G. M. (2007). The psychology of risk. Cambridge : Cambridge University Press.

- Calvez, M. (2000). La liminalité comme cadre d'analyse du handicap. Prévenir, 39, 83-89.

- Damour, C., \& Le Garrec, S. (2007). Perceptions des alcools et des alcoolisations chez les 25-35 ans. Principes de justice et rapports aux boires. Les cahiers de l'IREB, 18, 123-128.

- Dany, L. (2014, sous presse). La prévention entre continuité et changements : réflexions psychosociales. In : M. Saint-Jean \& C. Eymard (Eds.). Du changement dans le champ de la santé. Bruxelles : De Boeck-Estem.

- Dany, L., \& Apostolidis, T. (2002). L'étude des représentations sociales de la drogue et du cannabis : un enjeu pour la prévention. Santé publique, 14, 335-344.

- Dany, L., \& Apostolidis, T. (2012). Pensée sociale et risques dans le domaine de la santé : le regard des représentations sociales. Psychologie française, 87, 67-81.

- Demeulenaere, P. (2003). Les normes sociales. Entre accords et désaccords. Paris : PUF.

- De Chazeron, I., Llorca, P. M., Ughetto, S., Vendittelli, F., Boussiron, D., Sapin, V., Coudore, F., $\&$ Lemery, D. (2008). Is Pregnancy the Time to Change Alcohol Consumption Habits in France?. Alcoholism: Clinical and Experimental Research, 32, 868-873.

- Dumas, A., Simmat-Durand, L., \& Lejeune, C. (2014, sous presse). Grossesse et usage de substances psychoactives en France. Synthèse de la littérature. Journal de Gynécologie Obstétrique et Biologie de la Reproduction. http://dx.doi.org/10.1016/j.jgyn.2014.05.008

- Famy, C., Streissguth, A. P., \& Unis, A. S. (1998). Mental illness in adults with fetal alcohol syndrome or fetal alcohol effects. American Journal of Psychiatry, 155, 552-554.

- Fassin, D., \& Memmi, D. (2004). Le gouvernement des corps, cas de figure. Paris : Editions de l'EHESS.

- Fillmore, K. M. (1987). Women's drinking across the adult life course as compared to men's. British Journal of Addiction, 82, 801-811.

- Flament, C. (1994). Aspects périphériques des représentations sociales. In C. Guimelli (Ed.). Structures et transformations des représentations sociales (pp. 85-118). Lausanne : Delachaux et Niestlé.

- Gaussot, L. (2004). Modération et sobriété : Etudes sur les usages sociaux de l'alcool. Paris : L'Harmattan.

- Guillemont, J., \& Léon, C. (2008). Alcool et grossesse : connaissances du grand public en 2007 et évolutions en trois ans. Inpes, Évolutions, 15, 1-6.

- Hyman, H. H. (1942). The psychology of status. Archives of psychology, 269.

- INPES. (2006). Zéro alcool pendant la grossesse. Dossier de presse. Saint-Denis : INPES 
— INSERM. (2001). Alcool : Effets sur la santé. Expertise collective. Paris : Inserm.

- IREB. (2010). Observatoire 2010 : Les Français et l'alcool. Dossier de presse IREB. Paris: IREB.

- Jodelet, D. (2006). Place de l'expérience vécue dans les processus de formation des représentations sociales. In V. Haas (Ed), Les savoirs du quotidien (pp. 235-255). Rennes : PUR.

— Jodelet, D. (1989). Les représentations sociales. Paris : PUF.

- Jacobson, J. L., \& Jacobson, S. W. (2002). Effects of prenatal alcohol exposure on child development. Alcohol Research and Health, 26, 282-286.

- Kelly, S. J., Day, N., \& Streissguth, A. P. (2000). Effects of prenatal alcohol exposure on social behavior in humans and other species. Neurotoxicology and Teratology, 22, 143-149.

- Lapierre, S., \& Damant, D. (2012) Regards critiques sur la maternité dans divers contextes sociaux. Québec : Presses de l'Université du Québec.

- Lamblin, D. (2005). Les enfants porteurs de l'ensemble des troubles causés par alcoolisation foetale et leur devenir. In Alcool, grossesse et santé des femmes, Lille : Comité Départemental de Prévention, 57-61.

- Lo Monaco, G., Guimelli, C., \& Hidalgo, M. (2010). Contextes et normes dans la perception de l'alcoolisme. Alcoologie et Addictologie, 32, 45-51.

— Lupton, D. (1999). Risk. London : Routledge.

- Maalouf, D., El Hachem, H., Kesrouani, A., Hleis, S., Rohayem, J., Chammai, R., Hadadd, G., Haddad, R., \& Richa, S. (2011). Les conduites d'alcoolisation des femmes enceintes libanaises et leurs connaissances sur les risques et répercussions sur l'enfant à naître. L'Encéphale, 37, 94-100.

- Maisonneuve, J. (2000). Introduction à la psychosociologie. Paris : PUF.

- Maresca, B., Le Queau, P., Badeyan, G., \& Rotbart, G. (2000). Les attitudes vis-à-vis de l'alcool et du tabac après la loi Evin. Etudes et résultats, 78.

- Mattson, S. N., \& Riley, E. P. (1999). Implicit and explicit memory functioning in children with heavy prenatal alcohol exposure. Journal of the International Neuropsychological Society, 5, 46247.

- Meidani, A., Dany, L., \& Welzer-Lang, D. (2005). Manières de boire et rapports sociaux de genre chez les jeunes. Les cahiers de l'IREB, 17, 67-70.

- Menuel, J. (2011). Devenir enceinte. Socialisation et normalisation pendant la grossesse : Processus, réceptions, effets. Dossier d'études, 148. Paris: Caisse Nationale des Allocations Familiales.

- Morin, M. (1996). Perspectives de recherches pour l'étude empirique de l'explication sociale des maladies. Psychologie Française, 41, 147-154.

- Peretti-Watel, P. (2005). Cannabis, ecstasy : du stigmate au déni. Paris : Harmattan.

- Rainaut, J. (2000). La femme et l'alcool, quelques idées reçues. In C. Bernand (Ed). Désirs d'ivresse (pp. 463-489). Paris : Autrement.

- Rateau, P. (2000). L'approche structurale des représentations sociales. In N. Roussiau (Ed.), Psychologie Sociale (pp. 82-88). Paris : In-Press.

- Ricciardelli, L. A., Connor, J. P., Williams, R. J., Young, R. M. (2001). Gender stereotypes and drinking cognitions as indicators of moderate and high risk drinking among young women and men. Drug and Alcohol Dependence, 61, 129-136.

- Roebuck, T. M., Mattson, S. N., \& Riley, E. P. (1998). A review of the neuroanatomical findings in children with fetal alcohol syndrome or prenatal exposure to alcohol. Alcoholism: Clinical and Experimental Research, 22, 339-344. 
- Roebuck, T. M., Mattson, S. N., \& Riley, E. P. (1999). Behavioral and psychosocial profiles of alcohol-exposed children. Alcoholism: Clinical and Experimental Research, 23, 1070-1076.

- Santiago-Delefosse, M., \& Rouan, G. (2001). Méthodes qualitatives en psychologie. Paris : Dunod.

- Streissguth, A. P., Sampson, P. D., Olson, H. C., Bookstein, F. L., Barr, H. M., Scott, M., Feldman, J., \& Mirsky, A.F. (1994). Maternal drinking during pregnancy: attention and short-term memory in 14-year-old offspring. A longitudinal prospective study. Alcoholism: Clinical and Experimental Research, 18, 202-218.

- Toutain, S. (2009). Ce que les femmes disent de l'abstinence pendant la grossesse en France. Alcoologie et Addictologie, 31, 107-114.

- Thomas, S. E., Kelly, S. J., Mattson, S. N., \& Riley, E. P. (1998). Comparison of social abilities of children with fetal alcohol syndrome to those of children with similar IQ scores and normal controls. Alcoholism: Clinical and Experimental Research, 22, 528-533.

- Van Gennep, A. (1909/1981). Les rites de passage. Paris : Picard.

- Waterson, E. J., \& Murray-Lyon, I. M. (1989). Screening for alcohol related problems in the antenatal clinic: an assessment of different methods. Alcohol, 24, 21-30.

- Wilsnack, R. W., Wilsnack, S. C. \& Klassen, A. D. (1984). Women's Drinking and Drinking Problems: Patterns from a 1981 National Survey. American Journal of Public Health, 74, 12311238. 
Tableau 1 : Guide d'entretien

\begin{tabular}{|c|c|}
\hline Thèmes & Objectifs \\
\hline La grossesse & Questionner le vécu de la grossesse \\
\hline Changement d'habitudes & $\begin{array}{l}\text { Changements d'habitudes mis en place volontairement ou non, au } \\
\text { cours de la grossesse }\end{array}$ \\
\hline $\begin{array}{l}\text { Consommation d'alcool avant et au } \\
\text { cours de la grossesse }\end{array}$ & $\begin{array}{l}\text { Mise en place ou non d'une attention particulière de la } \\
\text { consommation d'alcool pendant la grossesse. }\end{array}$ \\
\hline $\begin{array}{l}\text { Rôle de l'entourage et des } \\
\text { professionnels quant à l'alcoolisation } \\
\text { pendant la grossesse }\end{array}$ & $\begin{array}{l}\text { Appropriation des messages de prévention véhiculés par les } \\
\text { professionnels de santé et l'entourage. L'alcoolisation pendant la } \\
\text { grossesse est-il un sujet traité }\end{array}$ \\
\hline Impact des messages de prévention & $\begin{array}{l}\text { Appropriation des messages de prévention véhiculés dans le cadre } \\
\text { des actions de santé publique }\end{array}$ \\
\hline Rapport aux professionnels de santé & $\begin{array}{l}\text { Identifier les freins à la déclaration des pratiques d'alcoolisation } \\
\text { de façon générale et dans le contexte du suivi médical de la } \\
\text { grossesse }\end{array}$ \\
\hline Représentations associées à l'alcool & $\begin{array}{l}\text { Etudier les représentations de l'alcool et de l'alcoolisation dans } \\
\text { le contexte de la grossesse chez des femmes enceintes. }\end{array}$ \\
\hline $\begin{array}{l}\text { Risques d'une alcoolisation au cours } \\
\text { de la grossesse }\end{array}$ & $\begin{array}{l}\text { Connaissance de la femme des risques d'une alcoolisation au } \\
\text { cours de la grossesse }\end{array}$ \\
\hline
\end{tabular}


Tableau 2 : Caractéristique de l'échantillon $(\mathrm{N}=64)$

N (\%)

Fréquence de la consommation d'alcool

Jamais

$37(57,8)$

1 fois par mois ou moins

$12(18,8)$

2 à 4 fois par mois

$1(15,6)$

2 à 3 fois par semaine

$4(6,2)$

4 fois ou plus par semaine

$1(1,6)$

Nombre de verres consommés par jour de consommation d'alcool

Aucun

$39(60,9)$

1 ou 2

$15(23,4)$

3 ou 4

$8(12,5)$

5 ou 6

$1(1,6)$

7 à 9

$1(1,6)$

Fréquence de consommation de plus de 6 verres en une seule occasion Jamais

Une fois par mois ou moins

4 fois ou plus par semaine

\section{DEPUIS LA GROSSESSE}

Fréquence de la consommation d'alcool

Jamais

1 fois par mois ou moins

Nombre de verres consommés par jour de consommation d'alcool

Aucun

1 ou 2

Consommation envisagée après la grossesse

Identique à avant la grossesse

Plus modérée qu'avant la grossesse

Plus excessive qu'avant la grossesse

Actuellement en couple

Oui

Non

Données manquantes

Niveau d'étude

Collège, BEP/CAP ou inférieur

Supérieur

$21(32,8)$

Donnée manquante

\section{CSP}

Artisans, commerçants, chef d'entreprise

Cadres, professions intellectuelles supérieures

Autres personnes sans activité

$34(53,1)$

Employés

$19(29,7)$

Professions intermédiaires

$4(6,3)$

Lieu de vie

Marseille

Hors Marseille

Données manquantes

Durée Grossesse

Premier trimestre

Deuxième trimestre

Troisième trimestre

Données manquantes

Primipare

Oui

Non

Revenus du foyer

Moins de $1000 €$

Entre 1000 et $1500 €$

Entre 1500 et $2000 €$

Entre 2000 et $2500 €$

$5(7,8)$

Plus de $2500 €$

$14(21,9)$


${ }^{1}$ La France occupe le cinquième rang des pays consommateur d'alcool (basé sur la consommation annuelle d'alcool pur/habitant de 15 ans et plus) (Direction de la Recherche des Études de l'Évaluation et des Statistiques, DREES).

${ }^{2}$ Un arrêté public du ministère de la santé et des Solidarités au J.O du 2 octobre 2006 (article L.3322-2 du code de la santé publique du 11 février 2005) précise que les bouteilles de boissons alcoolisées devront désormais être étiquetées, soit d'un pictogramme, soit d'un message sanitaire prévenant les femmes, mais également tout un chacun, des risques consécutifs à la consommation d'alcool pendant la grossesse. L'objectif est de permettre une meilleure information sur les risques sanitaires causés par la consommation d'alcool sur le fœetus pendant la grossesse, et notamment d'informer du risque encouru dès le premier verre d'alcool consommé par la mère. L'INPES, par cette campagne, rappelle qu'une consommation d'alcool même ponctuelle ou modérée pendant la grossesse, n'est pas anodine et peut entrainer des risques importants pour l'enfant à naître.

${ }^{3}$ «Par mises en représentation nous entendons l'idée que le risque est une construction sociale qui implique un ensemble d'enjeux à la fois individuels et sociaux qui, d'une part, s'actualise dans et par les situations auxquelles sont confrontés les individus et les groupes et, d'autre part, mobilise des cadres de pensée " déjà-là », préexistants au sein de la structure sociale. À ce titre, la construction du risque traduit un lien dynamique entre le sujet (individuel et collectif) et l'objet. Cette construction s'élabore dans et par des faits d'inscription et de participation sociales. L'objet constitue un support d'élaboration, d'actualisation, de confrontation et d'expression des formes de connaissances visant à le circonscrire et à le maîtriser » (Apostolidis \& Dany, 2012, p. 71).

${ }^{4}$ (Numéro d'entretien, âge, statut civil, primiparité, période de grossesse, statut de consommatrice (une consommatrice est quelqu'un qui boit au moins au cours des occasions)

${ }^{5}$ La notion de liminalité trouve son origine dans l'analyse des rites de passage (Van Gennep, 1909). « Elle qualifie le moment où un individu a perdu un premier statut et n'a pas encore accédé à un second statut ; il est dans une situation intermédiaire et flotte entre deux états » (Calvez, 2000).

${ }^{6}$ Comme le développe Memmi on fait face « à un gouvernement s'appuyant sur les sujets, c'est-à-dire qui confie volontiers la gestion rationnelle de l'objet dont il entend réguler les usages (ici le corps) aux individus, qui évalueront eux-mêmes l'évolution probable de leurs données corporelles et feront eux-mêmes le calcul des risques et des coûts financiers de sa correction. Ce gouvernement contemporain des conduites a pris pour nom: la biopolitique déléguée » (2004, p. 137). 